Genomic Epidemiology of VREfm in Victoria

\title{
The changing landscape of VREfm in Victoria, Australia: a State-wide genomic
}

\section{snapshot}

Robyn S. Lee ${ }^{\text {a\# }}$, Anders Gonçalves da Silva ${ }^{\mathrm{a}}$, Sarah L. Baines ${ }^{\mathrm{b}}$, Janet Strachan ${ }^{\mathrm{a}}$, Susan Ballard $^{\mathrm{a}}$, Glen P. Carter ${ }^{\mathrm{a}}$, Jason C. Kwong ${ }^{\mathrm{b}}$, Mark Schultz ${ }^{\mathrm{a}}$, Dieter Bulach ${ }^{\mathrm{a}}$, Torsten Seemann $^{\mathrm{c}}$, Timothy P. Stinear ${ }^{\mathrm{b}}$ and Benjamin P. Howden ${ }^{\text {a\# }}$

\author{
\#Address correspondence to: \\ Dr. Robyn Lee $\mathrm{PhD} \quad$ robyn.s.c.lee @ gmail.com ${ }^{1}$ \\ Prof. Benjamin Howden MD PhD bhowden@unimelb.edu.au \\ Type of manuscript: Research Article \\ Word count: Abstract 247, Intro 510, Results and Discussion 2,501 \\ Main tables: 1 \\ Main figures: 4 \\ Key words: Enterococcus; Genomic epidemiology; antimicrobial resistance; transmission; \\ infection control
}

\footnotetext{
${ }^{1}$ Robyn Lee is currently at the Center for Communicable Disease Dynamics, at the Harvard T.H. Chan School of Public Health, 677 Huntington Avenue, Boston, MA, USA 02115
} 
Genomic Epidemiology of VREfm in Victoria

31 Abstract (247/250 words)

32 Vancomycin-resistant Enterococcus faecium (VREfm) represent a major source of

33 nosocomial infection worldwide. In Australia, the vanB genotype is dominant; however there

34 has been a recent increase in the predominantly plasmid-encoded vanA genotype, prompting

35 investigation into the genomic epidemiology of VREfm in this context.

\section{Materials and Methods.}

37 A cross-sectional study of VREfm in Victoria, Australia (Nov.10 ${ }^{\text {th }}-$ Dec. $\left.9^{\text {th }}, 2015\right)$. A total of

38321 VREfm isolates (from 286 patients) were collected and whole-genome sequenced with

39 Illumina NextSeq. Single nucleotide polymorphisms (SNPs) were used to assess relatedness.

40 Multi-locus sequence types (STs), and genes associated with resistance and virulence were

41 identified. The vanA-harbouring plasmid from an isolate from each ST was assembled using

42 long-read data.

43 Results.

44 vanA-VREfm comprised $17.8 \%$ of isolates. ST203, ST80 and a pstS(-) clade, ST1421,

45 predominated $(30.5 \%, 30.5 \%$ and $37.2 \%$ of $v a n A$-VREfm, respectively). Most vanB-VREfm

46 were ST796 (77.7\%). vanA-VREfm isolates were closely-related within hospitals vs. between

47 them (core SNPs 10 [interquartile range 1-357] vs. 356 [179-416] respectively), suggesting

48 discrete introductions of vanA-VREfm, with subsequent intra-hospital transmission. In

49 contrast, $v a n B$-VREfm had similar core SNP distributions within vs. between hospitals, due

50 to widespread dissemination of ST796. Overall, vanA-harbouring plasmids differed across

51 STs, and with exception of ST78 and ST796, Tn1546 transposons also varied.

\section{Conclusions.}

53 vanA-VREfm in Victoria is associated with multiple STs, and is not solely mediated by a

54 single shared plasmid/Tn1546 transposon; clonal transmission appears to play an important 
bioRxiv preprint doi: https://doi org/101101/289975; this version posted March 28, 2018. The copyright holder for this preprint (which was not certified by peer review) is the author/funder, who has granted bioRxiv a license to display the preprint in perpetuity. It is made available under aCC-BY-NC-ND 4.0 International license.

Genomic Epidemiology of VREfm in Victoria

55 role, predominantly within, rather than between, hospitals. In contrast, vanB-VREfm appears

56 to be well-established and widespread across Victorian healthcare institutions.

57 
Genomic Epidemiology of VREfm in Victoria

58

\section{Introduction}

Enterococcus faecium is a leading cause of nosocomial infections world-wide $(1,2)$.

Resistance to glycopeptides, especially vancomycin, is the most clinically-relevant for $E$. faecium (1); mortality associated with any E. faecium blood-stream infection (BSI) exceeds 30\% (3), while patients with vancomycin-resistant E. faecium (VREfm) bacteremia have 2.5-fold higher odds of death compared to those with vancomycin-susceptible infection (4). Resistance to vancomycin in $E$. faecium can be conferred by different van gene clusters $(A, B$, $D, E, G, L, M$, and $N(5-8)$ ), with most infection attributed to vanA and vanB genotypes (9). VREfm is thought to be transmitted between hospitalized patients, healthcare staff, and/or via the hospital environment (10), with risk of colonization highest among those receiving long courses of antibiotics, the critically-ill and/or immunosuppressed, and those with prolonged hospitalization or history of nursing home residence (11). VREfm has also been shown to arise de novo in a patient, following transfer of vanB gene cluster from commensal gut anaerobes to E. faecium $(12,13)$.

The prevalence of the $v a n A$ and $v a n B$ genotypes varies geographically; in North America, vanA has been shown to drive the VREfm epidemic (e.g., (14)), while in Europe, both $v a n A$ and $v a n B$ genotypes play a role (15). In Australia, the first case of VREfm detected in 1994 carried the vanA operon (16); however, it is vanB-VREfm that has subsequently predominated.

Multiple clones of vanB-VREfm have been detected in Australia, mostly from sequence types (STs) within the hospital-associated clonal complex (CC) 17(9, 17-20). A survey of sentinel laboratories from across Australia in 2011 found that $97.2 \%(104 / 107$ isolates) of vancomycin-nonsusceptible isolates had the $v a n B$ genotype (17), over half of which were ST203 $(19,21)$. Subsequent country-wide surveys showed this strain dominated 
Genomic Epidemiology of VREfm in Victoria

82 until 2014, when it was surpassed by the ST796 clone (22) - largely due to its widespread

83 dissemination throughout Victoria and southeastern Australia (23).

84 Along with the spread of the vanB-associated ST796 clone, there has been a recent,

85 dramatic increase in vanA-VREfm; since 2011, the prevalence of vanA-VREfm bacteremia,

86 as measured by these sentinel surveys, increased from $<1 \%(2 / 341)$ to $21 \%(90 / 408$ isolates

87 genotyped) in 2016 (24). The reasons for this increase remain unclear, but multiple STs

88 appear to be involved. A retrospective study examining vanA-VREfm (screening and clinical

89 isolates) across four Australian hospitals from 2011-2013 (25) suggested this increase could

90 not be attributed to a single ST or vanA-harbouring plasmid; however, this was based on a

91 convenience sample of only 18 isolates from across the whole country, making this

92 inconclusive.

93 To further explore the potential reasons for the shift in van genotype, and increase our

94 understanding of the molecular epidemiology of VREfm, we conducted a population-level

95 study of VREfm in the State of Victoria - the second most inhabited State in Australia

96 (http://www.abs.gov.au/AUSSTATS/). All VREfm isolates from colonization and clinical

97 infection detected across the State over a month were subjected to whole genome sequencing,

98 as well as isolates from VSEfm bacteremia. In doing so, we provide an in-depth assessment

99 of the genomic diversity of E. faecium and novel insights into the epidemiology of VREfm in

100 this region.

101

102 Materials and Methods

103 Study design and population.

104 A cross-sectional survey of VREfm was conducted between November $10^{\text {th }}-$ December $9^{\text {th }}$,

1052015 in the State of Victoria (population: 5,931,100 for 2015, per the Australian Bureau of

106 Statistics; http://www.abs.gov.au/AUSSTATS/). During this period, all VREfm-positive 
Genomic Epidemiology of VREfm in Victoria

107 isolates (including screening and clinical samples) from all laboratories across the State were

108 sent to the Microbiological Diagnostic Unit Public Health Laboratory (MDU), as well as all

109 vancomycin-susceptible E. faecium isolated from blood cultures. Final lists of isolates

110 received by the MDU were cross-checked with primary diagnostic laboratories to ensure all

111 eligible isolates were included.

\section{DNA extraction and WGS.}

113 Genomic DNA was isolated from a single colony using a JANUS Chemagic Workstation and

114 Chemagic Viral DNA/RNA kit (PerkinElmer). Libraries were prepared with the Nextera XT

115 DNA sample preparation kit (Illumina). Whole genome sequencing (WGS) was performed

116 using the Illumina NextSeq 500 with 150 base-pair paired-end reads.

\section{Bioinformatics.}

118 Sequences were analysed using the Nullarbor pipeline (Seemann T, available at:

119 https://github.com/tseemann/nullarbor).

120 In brief, Illumina reads were trimmed for quality using Trimmomatic (v.0.36, (26)).

121 Contamination was assessed using Kraken (v.0.10.5, (27)). Reads were assembled into

122 contigs using SPAdes (v.3.10.1, (28)). In silico identification of resistance genes was done

123 with ABRicate (Seemann T, available at: https://github.com/tseemann/abricate), wherein the

124 assembled contigs were searched for resistance genes in the ResFinder database using

125 BLAST+ (29). Putative virulence genes were also identified in the same manner using the

126 Virulence Factor DataBase (30), to assess whether increased virulence could be responsible

127 for the rise in VREfm bacteremia in Australia. All positive hits were reviewed for gene

128 completeness (i.e., gene coverage and gaps), percent identity, and depth of coverage.

129 BLAST+ (29) was also used to search the assembled contigs for E. faecium alleles listed in

130 the pubMLST database (https://pubmlst.org); unknown allelic combinations and allelic

131 variants were submitted to the database for new assignment. Single nucleotide polymorphism 
Genomic Epidemiology of VREfm in Victoria

132 (SNP)-based analyses were done by aligning reads using the Burrows-Wheeler Aligner MEM

133 algorithm (v.0.7.16, (31)) to a novel local Victorian reference (ST796, AUSMDU00004028;

134 see Table A1 for details and metrics). This reference was selected because preliminary

135 analyses indicated the majority of our samples were also ST796. A minimum mapping

136 quality of 60 was required, and ambiguously-mapped reads were excluded. SNPs were called

137 using Freebayes (v1.0.2, (32)) under a haploid model, with a minimum depth of coverage of

13810 and allelic frequency of 0.9 required to call a SNP. Integrative Genomics Viewer

139 (v.2.3.88) was used to review alignments and SNPs as needed (33).

\section{Phylogenetics and population structure.}

141 ClonalFrameML was used to identify potential recombination (v.1.11, (34)). Putative

142 recombination sites were then masked using a custom script (available at:

143 https://github.com/kwongj/cfml-maskrc), and concatenated core SNP sites were extracted

144 using snp-sites -c (35). A maximum likelihood tree was then produced using RAxML

145 (v.8.2.11, (36)) under a General Time Reversible model with four gamma categories. One

146 thousand bootstrap replicates were performed to assess confidence in the tree. Figures were

147 produced using ggtree (37) in R (v.3.3.2). Bayesian Analysis of Population Structure with

148 hierarchical model-based clustering (hierBAPS; v.6.0, (38)) was also used to identify major

149 clades, using the alignments of recombination-adjusted core SNPs as input.

\section{Comparative genomics of vanA-VREfm.}

151 One strain from each of the major vanA-harbouring STs was sequenced using PacBio Single

152 Molecule, Real-Time sequencing with P6-C4 chemistry (Pacific Biosciences, CA). De novo

153 assembly was done using Canu (v.1.3 (39)); see Appendix for further detail. QUAST (v.4.5)

154 was used for additional quality metrics (40). vanA-harbouring plasmids from each of these

155 strains were identified using ABRicate, and subsequently annotated using Prokka (v. 1.11,

156 (41)) with the Enterococcus database 
Genomic Epidemiology of VREfm in Victoria

157 (https://github.com/tseemann/prokka/blob/master/db/genus/Enterococcus). To compare the

158 gene content of $v a n A$-harbouring plasmids, these plasmids were aligned to one another using

159 progressiveMauve (build date 2014-12-19) and visualized in Geneious (v.9.1.7, (42)).

160 Tn1546 transposons were identified and genes were manually compared across plasmids.

161 Victorian isolates in global context.

162 To assess how Victorian E. faecium strains fit in the global context, we re-ran our analyses

163 including 877 additional strains from Europe (872), North America (2), Africa (2) and Asia

164 (Israel; 1) (43-46), and isolates from the Welcome Trust Sanger BSAC Resistance

165 Surveillance Project (from NCBI BioProject PRJEB344). A maximum likelihood tree was

166 produced using IQ-TREE (v.1.6.1, (47); see Appendix) with model selection based on the

167 lowest Bayesian Information Criterion.

168 Statistical analysis.

169 Pairwise SNP distributions were compared using the Mann-Whitney rank-sum test.

170 Differences in proportion were compared using either the Chi-Square test or Fisher's Exact

171 test, as appropriate. Analyses were done in Stata (v.14.2, College Station, TX: StataCorp).

172 Data availability.

173 Illumina data, and the PacBio reads with corresponding assemblies for the five isolates listed

174 in Table A1 are available on the National Center for Biotechnology Information's (NCBI)

175 Sequence Read Archive under BioProject PRJNA433676.

176

177 Results

178 Between November $10^{\text {th }}-$ December $9^{\text {th }} 2015,333$ E. faecium-positive samples were

179 detected at 19 primary diagnostic laboratories from across Victoria (two additional

180 laboratories reported no E. faecium during this time). Two eligible VSEfm isolates were not 
Genomic Epidemiology of VREfm in Victoria

181 submitted, thus 331 isolates were available for sequencing. All isolates passed WGS quality

182 control (see the Appendix for details).

183 In total, 321 isolates (from 286 patients) were VREfm - 20 of which were from BSI

184 (from 17 patients). Ten VSEfm isolates (from ten patients) were also collected; one of these

185 was likely a false negative, as the patient also had a vanA-VREfm isolated from blood

186 collected the same day (further detail in the Appendix). Thus, 27 patients had E. faecium

187 bacteremia over this period; an incidence of 5.5/100,000 when extrapolated to one year.

188 Most patients ( $\mathrm{n}=257,87.1 \%$ ) were from hospitals in major metropolitan centers,

189 compared to rural areas $(n=29,9.8 \%)$, consistent with the geographical distribution of the

190 population in this State. Overall, 35 hospital networks (HCNs) were represented, with isolates

191 received from up to five affiliated hospitals from a single network. Five isolates were

192 collected in community medical/dental clinics (3.1\%). Sex and age were not available for

193 patients from $\operatorname{HCN} 1(n=61,20.7 \%)$; among the remaining patients, 113 were male (48.3\%),

194 and the median age was 71 (range 18-98, IQR 58-80). At sample collection, 256 persons

195 were inpatients and 36 were outpatients. Four patients were missing location of isolation and

196 had unknown admission status.

197 Twenty-seven patients had >1 sample positive for E. faecium (range 2-5 per patient;

198 Table A2), with a maximum of 40 SNPs between repeat isolates of the same ST (Figure

199 A1). Repeat samples were collected within the same hospital as the original sample for $26 / 27$

200 patients $(96.3 \%$, Table A2).

201 Identification of van genotype.

202 In silico genotyping revealed that $260(78.6 \%)$ of isolates were exclusively vanB, and 59

$203(17.8 \%)$ were exclusively vanA. There was no association between van genotype and BSI

204 (Figure 1, Fisher's Exact p=0.52). Two isolates were positive for $\operatorname{vanA}$ and $\operatorname{vanB}(<1 \%)$.

205 These were most closely related to $v a n B$-VREfm isolates, with as few as 0 pairwise core 
Genomic Epidemiology of VREfm in Victoria

206 SNPs compared to vanB-VREfm isolates (vs. 195 for vanA-VREfm), suggesting they already

207 had vanB when vanA was acquired. As expected, all isolates with vanA and/or vanB were

208 phenotypically resistant to vancomycin (MIC > $4 \mathrm{mg} / \mathrm{L}(48)$ ). Neither vanA or vanB were

209 found in isolates classified as VSEfm (3.0\%).

210 Overall, vanB-VREfm was more widely dispersed across Victorian hospitals and

211 HCNs compared to vanA-VREfm; excluding the two vanA+vanB-positive samples, vanB-

212 VREfm isolates were identified in 34/35 HCNs, while vanA-VREfm was only identified in

$21310 / 35$ during this time period $(\mathrm{p}<0.00005)$. VSEfm isolates were from six different

214 networks.

215 Multi-locus sequence typing.

216 Eighteen previously-known MLSTs and four novel STs were identified. Most isolates were

217 ST796 (61.3\%, Table 1). The pstS allele was missing for $22(6.6 \%)$ isolates; these were

218 classified as ST1421 based on remaining alleles (Table A3).

$219 v a n A$ was found in fewer STs than $v a n B$ (3 vs. 11, respectively, excluding $v a n A+B$

220 positive isolates), though this trend was not statistically significant (Fisher's Exact test,

$221 \mathrm{p}=0.17)$. vanA was almost exclusively present in ST203 and ST80, and ST1421 isolates

222 (Table 1). Most vanB-positive isolates were ST796.

223 Phylogenetics and population structure.

224 Prior to adjusting for recombination, there were a median of 20 core SNPs between study

225 isolates and the reference (IQR 14-2,653); adjusting for recombination reduced this to a

226 median of 12 core SNPs (IQR 9-238). An alignment of these adjusted core SNPs was used to

227 produce a maximum likelihood tree (Figure 2; recombination blocks are shown in Figure

228 A2). Many branches had very low bootstrap support, therefore BAPS was used to validate

229 clusters. 
Genomic Epidemiology of VREfm in Victoria

230 Five major BAPS groups were identified, which were largely consistent with the

231 phylogeny (Figure 2). BAPS-1 was comprised predominantly of ST796 isolates (203/214,

$23294.9 \%$ ), and was highly clonal, with a median of 10 core SNPs separating isolates (IQR 7-

233 15). In contrast, BAPS-2 was comprised of multiple STs. The highest proportions were from

234 ST203 (36/82, 43.9\%), ST1421 (22/82, 26.8\%) and ST17 (12/82, 14.6\%). Isolates in BAPS-2

235 were separated by a median of 257 core SNPs (IQR 167-312). Excluding repeat samples from

236 the same patient, 81 pairs of isolates were within one SNP. Sixty-six of these pairs (81\%)

237 were isolated at the same hospitals as one another $(\mathrm{H} 1, \mathrm{H} 3, \mathrm{H} 5)$, while the remainder were

238 predominantly from different HCNs (see Figure A3). BAPS-3 was comprised of a single

239 VSEfm isolate from ST54, which was over 3,900 SNPs from other isolates (not shown in

240 Figure 1). BAPS-4 isolates were predominantly from ST80 (25/31, 80.6\%), but also included

241 isolates from ST17, ST78 and ST262. Isolates were separated by a median of 252 core SNPs

242 (IQR 6-346). All pairs within one core SNP were from ST80; excluding repeat isolates, 8/15

243 of these pairs were collected at the same hospitals as one another (H1, H2 and H4). BAPS-5

244 was comprised of three VSEfm isolates (ST21, ST22, and ST32), with a minimum of 369

245 core pairwise SNPs.

246 Genetic diversity across hospitals and van genotypes.

247 Excluding comparisons within the same patient (shown in Figure A2), the median pairwise

248 core SNP distances among VREfm were similar within and between hospitals, at 196 (IQR

249 13-297) and 193 (IQR 13-207), respectively (Figure 3A).

250 As we hypothesized there were differences in diversity between isolates with different

251 VREfm genotypes, vanA-VREfm and vanB-VREfm were analyzed separately. vanA-VREfm

252 were significantly less diverse within hospitals compared to between them (Figure 3B,

$253 \mathrm{p}=0.0001$ ), suggesting multiple, independent introduction events with subsequent intra-

254 hospital transmission. In contrast, $v a n B$-VREfm was more diverse within hospitals vs. 
Genomic Epidemiology of VREfm in Victoria

255 between them (Figure 3C), p=0.0001), consistent with wide-spread dissemination and long-

256 term establishment of these strains across institutions within the Victorian healthcare system.

257 Virulence.

258 Isolates were interrogated for the presence of putative virulence factors; as shown in Figure

2592 , only two isolates (both ST17) had the cylA gene which is required for expression of

260 cytolysin (49). No genes encoding hyaluronidase $\left(h y l_{\mathrm{Efm}}(50)\right)$, agglutination substance

261 (agg/asal (51) or gelatinase (gelE (52)) were detected; the latter two genes are thought to be

262 more prevalent in E. faecalis than E. faecium (53).

263 Microbial surface components recognizing adhesive matrix molecules including acm

264 (54), which has been found predominantly in clinical isolates, as well as ecbA (55) and $\operatorname{sgrA}$

265 (55) were also investigated. acm was present in $98.5 \%$ of study samples, across nearly all

266 STs. $e c b A$ was present in 62 isolates (30.5\% of vanA-VREfm; $15.8 \%$ of vanB-VREfm),

267 including all ST192, ST400 and ST893 isolates, and most ST17 and ST203 isolates (71\% and

$26897 \%$, respectively). sgrA was present in 294 isolates (91.9\%, of vanA-VREfm; $89.2 \%$ of

$269 v a n B$-VREfm) from nearly all STs. No isolates had pilA and pilB, which are also potentially

270 involved in adhesion (56).

271 Finally, the gene esp, which has previously been associated with hospital outbreaks

272 (57) and encodes enterococcal surface protein (58), was investigated. While repeat regions

273 made it impossible to completely assemble esp using short-read data (see Appendix, Figure

274 A4), we found that 266 isolates had at least $80 \%$ coverage (81.6\% of vanA-VREfm; $86.2 \%$ of

$275 v a n B$-VREfm), suggesting that these isolates were likely esp-positive. In this context, esp was

276 more prevalent in bacteremia due to VREfm than VSEfm (17/20 vs. $1 / 9, p=0.0001$, excluding

277 the VSEfm that had likely lost the vanA operon). 
Genomic Epidemiology of VREfm in Victoria

278

279

280

281

282

283

284

285

The vanA-harbouring plasmid from one isolate per ST was completely assembled

using long-read data; no putative virulence genes were found to co-localize with vanA on the same plasmid (Figure A5).

\section{Comparative genomics of vanA-VREfm.}

The vanA-harbouring plasmids from ST78 and ST796 had the greatest homology (Figure 4), with similar plasmid backbones and $100 \%$ amino acid identity of the Tn1546 transposon, which carries the vanA gene cluster (Figure A5). The main difference between these plasmids was a large insertion in ST796 compared to ST78, carrying repB, IS1216 and two hypothetical proteins (Figure A5). The vanA-harbouring plasmid from ST80 was similar to that from ST796 (Figure A6), but had lost ISEfa7-birA from the plasmid backbone (Figure A5) and had a 48-bp deletion in vanS compared to the ST796 plasmid. Both vanA-harbouring plasmids from the ST203 and ST1421 isolates had unique plasmid backbones and Tn1546 transposons (Figure A5). Together, these findings suggest that horizontal transfer of a single vanA-harbouring plasmid and/or Tn1546 carrying the vanA gene cluster is not responsible for the increase in vanA-VREfm in Victoria.

\section{Victorian isolates in global context.}

Compared to published sequences, the vanA-harbouring plasmids from ST78, ST796 and ST80 shared closest homology to a vanA-harbouring plasmid from VREfm in Demark (46)

(Table A4). The vanA-harbouring plasmid from ST203 was most closely related to plasmids from several STs of VREfm isolated in Western Australia and Queensland (25), while the plasmid from ST1421 was closely-related to that from another ST1421 E. faecium, isolated in New South Wales in 2014 (59) (Table A4). Phylogenetic analysis based on core SNPs from Victorian isolates and global $E$. faecium strains showed that, with the exception of those from ST796, Victorian isolates were 
Genomic Epidemiology of VREfm in Victoria

302 widely distributed across the tree (Figure A7). This suggests ongoing, inter-continental

303 transmission of E. faecium.

304

\section{Discussion}

306 For the past two decades, the majority of VREfm in Australia have been vanB genotype (13,

307 18, 19, 21-23, 60-62). Recently, however, vanA-VREfm has been increasing. In this

308 population-based study, we looked at factors potentially contributing to this change in

309 epidemiology.

310 Firstly, we examined the genetic diversity of vanA-VREfm across our State, to assess

311 for potential transmission. Consistent with the hypothesis of van Hal et al. (25), we found that

312 a single clone is not driving this increase; instead, our findings suggest that multiple

313 introductions of vanA-VREfm have occurred, with subsequent spread within Victorian

314 hospitals. This pattern is similar to that of early vanB-VREfm in Australia (63), though the

315 latter has become dominated by a single ST in Victoria (ST796) (23).

316 Another possibility was that a single vanA-harbouring plasmid and/or Tn1546

317 transposon was being transmitted within and across different STs, as has been shown in

318 Denmark (46). As a unique vanA-harbouring plasmid was present in representatives from

319 each ST, and nearly all had different Tn1546 transposons, this suggests that horizontal gene

320 transfer is not the sole driver of $v a n A-V R E f m$ in Victoria.

321 Finally, we assessed whether there were differences in putative virulence factors

322 between our isolates, and those from other studies/settings. Overall, few putative virulence

323 factors were identified, and their prevalence was largely consistent with previous data from

$324 v a n B$-VREfm in Australia (64), and similar to or lower than those found in vanA-VREfm

325 from settings (e.g., in China (65) or Brazil (66)). While much is still unknown about

326 virulence factors in VREfm (compared to Enterococcus faecalis), this suggests the rise in 
Genomic Epidemiology of VREfm in Victoria

327 vanA-VREfm in Australia, and the continued success of vanB-VREfm, is not due to changes

328 in virulence. Consistent with this, the rate of $E$. faecium BSI in our study was similar to that

329 of another population-based study in Denmark (3). This has important implications for

330 hospital infection control; as the patient population affected by VREfm has also remained

331 consistent over time, we propose that environmental factors may be mediating the observed

332 changes in VREfm epidemiology. Further studies are needed to investigate this hypothesis.

333 In addition to gaining new insights in vanA epidemiology in this context, we have also

334 provided valuable information on $v a n B$-VREfm; given that we found genetic diversity was

335 slightly lower across hospitals compared to within, this suggests a potential community

336 reservoir for these strains. Transmission of VREfm has previously been shown in nursing

337 homes (67), with carriage strains closely related to those causing infection; investigation is

338 warranted to determine if this is also the case for Victoria. The seemingly widespread

339 diversity of vanB VREfm could also be due to a "diffusion" effect, wherein colonization and

340 infection have slowly spread across hospitals through many years of patient exchanges. Such

341 a pattern would be reminiscent of that observed in Denmark (68); however, while in

342 Denmark the "diffusion" process was marked by a hub-and-spoke pattern, with patients

343 mainly being exchanged between regional hospitals and central hospitals in Copenhagen, in

344 Victoria, the process was likely more random. If such a "diffusion" scenario is correct, we

345 hypothesize that the pattern of genetic diversity of vanA-VREfm will eventually mirror that

346 of the $v a n B$.

347 This work has a number of key strengths. Firstly, by including $>99 \%$ of VREfm

348 collected in Victoria over this one-month period, we have provided a comprehensive

349 assessment of local strain diversity and the prevalence of clinically-relevant van genotypes,

350 as well as a baseline to monitor for changes in VREfm population in Victoria. Secondly, we

351 used local reference genomes for all SNP-based analyses. As using more genetically-distant 
Genomic Epidemiology of VREfm in Victoria

352 reference genomes can result in false positive SNPs, and/or loss of information due to

353 differences in genes present in the study sample, but not in the reference, this increased the

354 accuracy of our short-read analyses. Since the first complete E. faecium genome was

355 published by our group in 2012 (69), few other genomes have been completed; by sharing

356 these complete VREfm genomes (from five different STs), we are also providing an

357 invaluable resource for public health as well as future studies on E. faecium. Finally, the use

358 of long-read data has also allowed us to completely assemble and characterize the vanA-

359 harbouring plasmids in each of the different STs - a task that is not always feasible with

360 short-read data alone.

361 There are several limitations of this work. Firstly, screening protocols for VREfm

362 colonization are not standardized across Victoria; each hospital adhered to its own infection

363 control guidelines, and as such, there may be incomplete capture of colonizing strains in this

364 dataset. This may bias our dataset somewhat towards strains causing clinical infection, and

365 cause us to miss potential transmission. Another limitation is that we did not have any data on

366 patient contact or intra-hospital transfer (except where isolates were collected at different

367 hospitals) - preventing us from confirming direct person-to-person transmission events.

368 However, we do not think this affects our overall population-level inferences, given the

369 dramatically lower genetic diversity of vanA within hospitals compared to vanB. Finally, as

370 this was predominantly a lab-based study, we also did not have data on clinical presentation

371 at time of sample collection and were therefore unable to discriminate clinical infection aside

372 from bacteremia. Thus, we may have underestimated the overall prevalence of infection

373 (though the most clinically important cases - associated with bacteremia - have been

374 included).

375 Herein, we have provided a comprehensive snapshot of VREfm strain diversity across

376 Victoria. We highlight the complexity of E. faecium genomic epidemiology, revealing key 
Genomic Epidemiology of VREfm in Victoria

377 differences in transmission dynamics of clinically-relevant van genotypes in this setting. Our

378 findings are applicable to not only to Victoria, but other regions with VREfm - particularly

379 those where the prevalence of $v a n B$ is high. While $v a n B$-VRE continues to predominate in

380 Victoria, repeated introductions and dissemination of vanA-VREfm within our hospitals

381 suggest this is changing. To better understand the clinical significance of this shift,

382 prospective studies with detailed corresponding clinical data are needed. As plasmids

383 carrying vanA or their Tn1546 transposons may be may be readily transferred to VSEfm

384 and/or rarely to other species (e.g., (70)), preventing an increase in vanA-VREfm is critical.

385 This reinforces the overall importance of ongoing prevention and control of VREfm.

387 Transparency. Authors have no conflicts of interest to declare.

389 Funding. RSL is supported by a Fellowship from the Canadian Institutes of Health Research

390 (Funding Reference Number 152448). SLB holds an Australian Government Research

391 Training Program Scholarship. JCK is supported by an early career fellowship from the

392 NHMRC (GNT1142613). BPH has a Practitioner Fellowship from the National Health and

393 Medical Research Council (NHMRC), Australia (GNT1105905), and is supported by the

394 Centre of Research Excellence on Emerging Infectious Diseases (NHMRC GNT1102962).

395 The Microbiological Diagnostic Unit Public Health Laboratory is funded by the Victorian

396 Government, Australia.

398 Acknowledgements. We would like to thank the participating laboratories for their provision

399 of samples and the clerical and technical assistance of staff at MDU PHL. We would also like

400 to thank Dr. Sharon Peacock (Department of Medicine, University of Cambridge, Cambridge,

401 United Kingdom; Wellcome Trust Sanger Institute, Hixton, United Kingdom; London School 
Genomic Epidemiology of VREfm in Victoria

402 of Hygiene and Tropical Medicine, London, United Kingdom) and Dr. Kathy Raven

403 (Department of Medicine, University of Cambridge, Cambridge, United Kingdom) for

404 helpful discussions about global VREfm.

405

406 Contributions. RSL designed and ran the primary analyses, interpreted results, made the

407 tables and figures, and wrote the first draft of the manuscript. AG designed analyses, advised

408 on bioinformatics approaches, helped interpret results, and contributed to writing the

409 manuscript. SLB assembled the PacBio genomes, provided input on analyses, and helped

410 interpret results. JS recruited all labs, coordinated submission of isolates and data from

411 primary diagnostic laboratories, and did the initial processing of samples at MDU PHL. SB

412 designed sample collection and advised on the initial interpretation of the results. GPC did

413 the laboratory work to generate the PacBio sequences. JCK, MS, DB and TS provided

414 bioinformatics tools, and advised on bioinformatics analyses. TPS and BPH conceived the

415 cross-sectional study, helped decide on analyses, and edited the manuscript. All authors

416 critically reviewed the manuscript for content. 
Genomic Epidemiology of VREfm in Victoria

418 Figure Legends.

Figure 1. Specimen type by van gene for Victorian $E$. faecium isolates

421 Only 2 isolates were vanA+vanB; only one was from a blood-stream infection (BSI). As all

42210 VSEfm isolates were from BSI by design, these have not been shown. Fisher Exact test

423 comparing the proportion of BSI among isolates with vanA vs vanB: $\mathrm{p}=0.52$.

425 Figure 2. Maximum likelihood tree of $E$. faecium in Victoria

426 6,497 recombination-adjusted core SNPs from all 331 isolates (321 VREfm and 10 VSEfm)

427 were concatenated, and used to produce a maximum likelihood tree in RAxML (36) under a

428 General Time Reversible model with gamma rate heterogeneity. One thousand bootstrap

429 replicates were performed to assess confidence in the phylogeny. BAPS-3 is not shown;

430 AUSMDU00004157 (an ST54 VSEfm) was the only isolate in this clade, and was >3,800

431 SNPs from all other isolates, so it was excluded from this figure for easier visualization.

432 Branches with bootstrap support >90 are shown. Many branches had very low bootstrap

433 support, thus hierarchical Bayesian Analysis of Population Structure (hierBAPS) was used to

434 further validate the clusters identified. Main BAPS groups are shown. The reference is

435 identified with an arrow. No isolates had $e b p A$, hyl, gelE, agg, pilA or pilB, therefore these

436 genes have not been shown.

438 Figure 3. Recombination-adjusted pairwise core SNPs separating Victorian isolates

439 A. Pairwise SNP comparisons are shown regardless of van gene. There were 54,567 total

440 pairwise comparisons, after excluding those involving isolates with missing data on

441 hospital/HCN, those from unknown clinics, and pairs within the same patients.

442 Between hospitals, the median pairwise SNPs was 193 (IQR 11-298), while within hospitals,

443 the median was 196 (IQR 13-297). For easier visualization, as one VSEfm isolate 
Genomic Epidemiology of VREfm in Victoria

444 (AUSMDU00004157) was a minimum of 3,893 SNPs from all others, only comparisons

445 between the 330 other isolates are shown in the figure.

446 B. Pairwise comparisons between isolates with vanA only are shown. There were 1,706 such

447 comparisons (233 within hospitals 1,473 between hospitals). The median pairwise SNPS

448 within hospitals was 10 (IQR 1-357) compared to a median of 356 (IQR 179-416) between

449 hospitals $(\mathrm{p}=0.0001)$.

450 C. Pairwise comparisons between isolates with vanB only are shown. There were 33,639

451 such comparisons (3,104 within hospitals, 30,528 between hospitals). The median pairwise

452 SNPs within hospitals was 40 (IQR 9-206), compared to a median of 15 (IQR 8-174)

453 between hospitals $(\mathrm{p}=0.0001)$.

454 Isolates with both $v a n A$ and $v a n B$-positive $(\mathrm{n}=2)$, and isolates that were $v a n A$ and $v a n B$ -

455 negative $(\mathrm{n}=10)$ were excluded from Panels B and C.

457 Figure 4. Homology between vanA-harbouring plasmids

458 PacBio Single Molecule Real-Time sequencing was performed on a vanA-positive isolate

459 from each of the main vanA-associated STs (ST 78, ST80, 203, 796, and ST1421). Plasmids

460 harbouring vanA were initially identified using ABRicate

461 (https://github.com/tseemann/abricate), and subsequently annotated using the Enterococcus

462 database (https://github.com/tseemann/prokka/blob/master/db/genus/Enterococcus). This

463 figure was produced using EasyFig (v. 2.2.2, version); BLASTn was used to compare

464 sequence homology. Only hits with a minimum length of $200 \mathrm{bp}$ and at least $99 \%$ identity are

465 shown for clarity. van genes are indicated in red, all other genes are indicated in blue.

466 


\section{Table 1. Multi-locus sequence types (MLSTs) identified}

\begin{tabular}{|c|c|c|c|c|c|}
\hline MLST & $\begin{array}{l}\text { Number of } \\
\text { isolates, } \mathrm{n}(\%)\end{array}$ & vanA only, $\mathrm{n}(\%)$ & vanB only, n (\%) & $\operatorname{van} A+B, \mathrm{n}(\%)$ & $\begin{array}{l}\text { Neither vanA nor } \\
\text { van } B, \mathrm{n}(\%)\end{array}$ \\
\hline 796 & $203(61.3)$ & & $202(77.7)$ & $1(50.0)$ & \\
\hline 203 & $37(11.2)$ & $18(30.5)$ & $19(7.3)$ & & \\
\hline 80 & $26(7.9)$ & $18(30.5)$ & $5(1.9)$ & & $3(30.0)^{d}$ \\
\hline 17 & $17(5.1)$ & & $15(5.8)$ & $1(50.0)$ & $1(10.0)$ \\
\hline 1421 (pstS(-)) & $22(6.6)$ & $22(37.2)$ & & & \\
\hline $1429^{a}$ & $5(1.5)$ & & $5(1.9)$ & & \\
\hline ?new ${ }^{b}$ & $1(0.3)$ & & $1(0.4)$ & & \\
\hline Other $^{c}$ & $20(6.0)$ & $1(1.7)$ & $13(5.0)$ & & $6(60.0)$ \\
\hline Total, n (\%) & 331 & $59(17.8)$ & $260(78.5)$ & $2(1.0)$ & $10(3.0)$ \\
\hline
\end{tabular}

$\mathrm{N}=331$ isolates. ${ }^{\mathrm{a}}$ A novel single-locus variant of ST796. ${ }^{\mathrm{b}}$ The $d d l$ gene might be interrupted in this isolate, but we were unable to resolve it using short-read data, and were therefore not able to type it. It clusters with ST17 isolates in the recombination-adjusted phylogenetic tree. ${ }^{\mathrm{c}}$ Sixteen additional STs were identified, each represented by three or fewer isolates. The single vanA isolate here corresponds to ST78. ${ }^{\mathrm{d}}$ This includes the one isolate that likely lost the vanA -harbouring plasmid during passage. 
Genomic Epidemiology of VREfm in Victoria

\section{References}

1. Gilmore MS, Lebreton F, van Schaik W. 2013. Genomic transition of enterococci from gut commensals to leading causes of multidrug-resistant hospital infection in the antibiotic era. Curr Opin Microbiol 16:10-6.

2. Guzman Prieto AM, van Schaik W, Rogers MR, Coque TM, Baquero F, Corander J, Willems RJ. 2016. Global emergence and dissemination of enterococci as nosocomial pathogens: attack of the clones? Front Microbiol 7:788.

3. Pinholt M, Ostergaard C, Arpi M, Bruun NE, Schonheyder HC, Gradel KO, Sogaard M, Knudsen JD, Danish Collaborative Bacteraemia. 2014. Incidence, clinical characteristics and 30-day mortality of enterococcal bacteraemia in Denmark 20062009: a population-based cohort study. Clin Microbiol Infect 20:145-51.

4. DiazGranados CA, Zimmer SM, Klein M, Jernigan JA. 2005. Comparison of mortality associated with vancomycin-resistant and vancomycin-susceptible enterococcal bloodstream infections: a meta-analysis. Clin Infect Dis 41:327-33.

5. Lebreton F, Depardieu F, Bourdon N, Fines-Guyon M, Berger P, Camiade S, Leclercq R, Courvalin P, Cattoir V. 2011. D-Ala-d-Ser VanN-type transferable vancomycin resistance in Enterococcus faecium. Antimicrob Agents Chemother 55:4606-12.

6. Depardieu F, Perichon B, Courvalin P. 2004. Detection of the van alphabet and identification of enterococci and staphylococci at the species level by multiplex PCR. J Clin Microbiol 42:5857-60.

7. Xu X, Lin D, Yan G, Ye X, Wu S, Guo Y, Zhu D, Hu F, Zhang Y, Wang F, Jacoby GA, Wang M. 2010. vanM, a new glycopeptide resistance gene cluster found in Enterococcus faecium. Antimicrob Agents Chemother 54:4643-7. 
Genomic Epidemiology of VREfm in Victoria

8. Courvalin P. 2006. Vancomycin resistance in gram-positive cocci. Clin Infect Dis 42 Suppl 1:S25-34.

9. Top J, Willems R, Bonten M. 2008. Emergence of CC17 Enterococcus faecium: from commensal to hospital-adapted pathogen. FEMS Immunol Med Microbiol 52:297308.

10. Ridwan B, Mascini, E., van der Reijden, N., Verhoef, J., Bonten, M. 2002. What action should be taken to prevent spread of vancomycin resistant enterococci in European hospitals? British Medical Journal 324:666-668.

11. Zirakzadeh A, Patel R. 2006. Vancomycin-resistant enterococci: colonization, infection, detection, and treatment. Mayo Clin Proc 81:529-36.

12. Stinear TP, Olden DC, Johnson PDR, Davies JK, Grayson ML. 2001. Enterococcal vanB resistance locus in anaerobic bacteria in human faeces. Lancet 357:855-856.

13. Howden BP, Holt KE, Lam MM, Seemann T, Ballard S, Coombs GW, Tong SY, Grayson ML, Johnson PD, Stinear TP. 2013. Genomic insights to control the emergence of vancomycin-resistant enterococci. MBio 4:e00412-3.

14. Simner PJ, Adam H, Baxter M, McCracken M, Golding G, Karlowsky JA, Nichol K, Lagace-Wiens P, Gilmour MW, Canadian Antimicrobial Resistance A, Hoban DJ, Zhanel GG. 2015. Epidemiology of vancomycin-resistant enterococci in Canadian hospitals (CANWARD study, 2007 to 2013). Antimicrob Agents Chemother 59:43157.

15. Werner G, Coque TM, Hammerum AM, Hope R, Hryniewicz W, Johnson A, Klare I, Kristinsson KG, Leclercq R, Lester CH, Lillie M, Novaris C, Olsson-Liljequist B, Leixe LV, Sadowy E, Simonsen GS, Top J, Vuopio-Varkila J, Willems RJ, Witte W, Woodford N. 2008. Emergence and spread of vancomycin resistance among enterococci in Europe. EuroSurveillance 13:1-11. 
Genomic Epidemiology of VREfm in Victoria

16. Kamarulzaman A, Tosolini, F.A., Boquest, A.L., Geddes, Richards, M.J. 1995.

Vancomycin-resistant Enterococcus faecium in a liver transplant recipient. Aust NZ J 25:560.

17. Coombs GW, Pearson JC, Le T, Daly DA, Robinson JO, Gottlieb T, Howden BP, Johnson PD, Bennett CM, Stinear TP, Turnidge JD, on behalf of the Australian Group on Antimicrobial Resistance. 2014. Australian Enterococcal Sepsis Outcome Progamme, 2011. Commun Dis Intell Q Rep 38:E247-52.

18. Christiansen K TJ, Gottlieb T, Coombs G, Bell J, George N, Pearson J. on behalf of the Australian Group for Antimicrobial Resistance. 2010. Antimicrobial susceptibility and VRE characterisation report of enterococcus isolates from the Australian Group on Antimicrobial Resistance (AGAR): 2010 Surveillance Report.

19. Coombs GW PJ, Daley DA, Le T, Robinson O, Gottlieb T, Howden BP, Johnson PDR, Bennett CM, Stinear TP, Turnidge J. on behalf of the Australian Group on Antimicrobial Resistance. 2013. Australian Group on Antimicrobial Resistance (AGAR) Australian Enterococcal Sepsis Outcome Programme (AESOP) Annual Report 2013.

20. Coombs GW, Pearson JC, Daley DA, Le T, Robinson OJ, Gottlieb T, Howden BP, Johnson PD, Bennett CM, Stinear TP, Turnidge JD, Australian Group on Antimicrobial R. 2014. Molecular epidemiology of enterococcal bacteremia in Australia. J Clin Microbiol 52:897-905.

21. Coombs GW Daley DA, Lee YT, Pang S, Pearson JC, Robinson O, Johnson PDR, Kotsanas D, Bell JM, Turnidge J. on behalf of the Australian Group on Antimicrobial Resistance. 2014. Australian Group on Antimicrobial Resistance (AGAR) Australian Enterococcal Sepsis Outcome Programme (AESOP) Annual Report 2014. 
Genomic Epidemiology of VREfm in Victoria

22. Coombs GW Daley DA. on behalf of the Australian Group for Antimicrobial Resistance GoAR. 2015. Australian Enterococcal Sepsis Outcome Program (AESOP) 2015 Final Report.

23. Buultjens AH, Lam MM, Ballard S, Monk IR, Mahony AA, Grabsch EA, Grayson ML, Pang S, Coombs GW, Robinson JO, Seemann T, Johnson PD, Howden BP, Stinear TP. 2017. Evolutionary origins of the emergent ST796 clone of vancomycin resistant Enterococcus faecium. PeerJ 5:e2916.

24. Coombs GW, Daley D, on behalf of the Australian Group for Antimicrobial Resistance. 2017. Australian Enterococcal Sepsis Outcome Program (AESOP) 2016.

25. van Hal SJ, Espedido BA, Coombs GW, Howden BP, Korman TM, Nimmo GR, Gosbell IB, Jensen SO. 2017. Polyclonal emergence of vanA vancomycin-resistant Enterococcus faecium in Australia. J Antimicrob Chemother 72:998-1001.

26. Bolger AM, Lohse M, Usadel B. 2014. Trimmomatic: a flexible trimmer for Illumina sequence data. Bioinformatics 30:2114-20.

27. Wood D, Salzberg, SL. 2014. Kraken: ultrafast metagenomic sequence classification using exact alignments. Genome Biol 15.

28. Bankevich A, Nurk S, Antipov D, Gurevich AA, Dvorkin M, Kulikov AS, Lesin VM, Nikolenko SI, Pham S, Prjibelski AD, Pyshkin AV, Sirotkin AV, Vyahhi N, Tesler G, Alekseyev MA, Pevzner PA. 2012. SPAdes: a new genome assembly algorithm and its applications to single-cell sequencing. J Comput Biol 19:455-77.

29. Zhang Z, Schwartz S, Wagner L, Miller W. 2000. A greedy algorithm for aligning DNA sequences. J Comput Biol 7:203-14.

30. Chen L, Zheng D, Liu B, Yang J, Jin Q. 2016. VFDB 2016: hierarchical and refined dataset for big data analysis--10 years on. Nucleic Acids Res 44:D694-7. 
Genomic Epidemiology of VREfm in Victoria

31. Li H. 2013. Aligning sequence reads, clone sequences and assembly contigs with BWA-MEM. arXiv:1303.3997v2.

32. Garrison E, Marth, G. 2012. Haplotype-based variant detection from short-read sequencing. arXiv:1207.3907v2.

33. Robinson JT, Thorvaldsdottir H, Winckler W, Guttman M, Lander ES, Getz G, Mesirov JP. 2011. Integrative genomics viewer. Nat Biotechnol 29:24-6.

34. Didelot X, Wilson DJ. 2015. ClonalFrameML: efficient inference of recombination in whole bacterial genomes. PLoS Comput Biol 11:e1004041.

35. Page AJ, Taylor, B., Delaney, A.J., Soares, J., Seemann, T., Keane, J.A., Harris, S.R. 2016. SNP-sites: rapid efficient extraction of SNPs from multi- FASTA alignments. Microb Genom doi:10.1099/mgen.0.000056.

36. Stamatakis A. 2014. RAxML version 8: a tool for phylogenetic analysis and postanalysis of large phylogenies. Bioinformatics 30:1312-3.

37. Yu G, Smith DK, Zhu H, Guan Y, Lam TT-Y, McInerny G. 2017. ggtree: anrpackage for visualization and annotation of phylogenetic trees with their covariates and other associated data. Methods Ecol Evol 8:28-36.

38. Cheng L, Connor TR, Siren J, Aanensen DM, Corander J. 2013. Hierarchical and spatially explicit clustering of DNA sequences with BAPS software. Mol Biol Evol 30:1224-8.

39. Koren S, Walenz BP, Berlin K, Miller JR, Bergman NH, Phillippy AM. 2017. Canu: scalable and accurate long-read assembly via adaptive k-mer weighting and repeat separation. Genome Res 27:722-736.

40. Gurevich A, Saveliev V, Vyahhi N, Tesler G. 2013. QUAST: quality assessment tool for genome assemblies. Bioinformatics 29:1072-5. 
Genomic Epidemiology of VREfm in Victoria

41. Seemann T. 2014. Prokka: rapid prokaryotic genome annotation. Bioinformatics 30:2068-9.

42. Kearse M, Moir R, Wilson A, Stones-Havas S, Cheung M, Sturrock S, Buxton S, Cooper A, Markowitz S, Duran C, Thierer T, Ashton B, Meintjes P, Drummond A. 2012. Geneious Basic: an integrated and extendable desktop software platform for the organization and analysis of sequence data. Bioinformatics 28:1647-9.

43. Lebreton F, van Schaik W, McGuire AM, Godfrey P, Griggs A, Mazumdar V, Corander J, Cheng L, Saif S, Young S, Zeng Q, Wortman J, Birren B, Willems RJ, Earl AM, Gilmore MS. 2013. Emergence of epidemic multidrug-resistant Enterococcus faecium from animal and commensal strains. MBio 4:e0534-13.

44. Raven KE, Gouliouris T, Brodrick H, Coll F, Brown NM, Reynolds R, Reuter S, Torok ME, Parkhill J, Peacock SJ. 2017. Complex Routes of Nosocomial Vancomycin-resistant Enterococcus faecium transmission revealed by genome sequencing. Clin Infect Dis 64:886-893.

45. Raven KE, Reuter S, Reynolds R, Brodrick HJ, Russell JE, Torok ME, Parkhill J, Peacock SJ. 2016. A decade of genomic history for healthcare-associated Enterococcus faecium in the United Kingdom and Ireland. Genome Res 26:13881396.

46. Pinholt M, Gumpert H, Bayliss S, Nielsen JB, Vorobieva V, Pedersen M, Feil E, Worning P, Westh H. 2017. Genomic analysis of 495 vancomycin-resistant Enterococcus faecium reveals broad dissemination of a vanA plasmid in more than 19 clones from Copenhagen, Denmark. J Antimicrob Chemother 72:40-47.

47. Nguyen LT, Schmidt HA, von Haeseler A, Minh BQ. 2015. IQ-TREE: a fast and effective stochastic algorithm for estimating maximum-likelihood phylogenies. Mol Biol Evol 32:268-74. 
Genomic Epidemiology of VREfm in Victoria

48. European Committee on Antimicrobial Susceptibility Testing. Breakpoint tables for interpretation of MICs and zone diameters, version 7.0. 2017. http://www.eucast.org/

49. Cox CR, Coburn, P.S., Gilmore, M.S. 2005. Enterococcal cytolysin: a novel two compotent peptide system that serves as a bacterial defense against eukaryotic and prokaryotic cells. Curr Protein \& Peptide Sci 6:77-84.

50. Rice LB, Carias L, Rudin S, Vael C, Goossens H, Konstabel C, Klare I, Nallapareddy SR, Huang W, Murray BE. 2003. A potential virulence gene, hylEfm, predominates in Enterococcus faecium of clinical origin. J Infect Dis 187:508-12.

51. Galli D, Lottspeich, F., Wirth, R. 1990. Sequence analysis of Enterococcus faecalis aggregation substance encoded by the sex pheromone plasmid pAD1. Mol Microbiol 4:1365-2958.

52. Su YA, Sulavik MC, He P, Makinen KK, Makinen PL, Fiedler S, Wirth R, Clewell DB. 1991. Nucleotide sequence of the gelatinase gene ( $\mathrm{gelE}$ ) from Enterococcus faecalis subsp. liquefaciens. Infect Immun 59:415-20.

53. Comerlato CB, de Resende MCC, Caierao J, d'Azevedo PA. 2013. Presence of virulence factors in Enterococcus faecalis and Enterococcus faecium susceptible and resistant to vancomycin. Memorias Do Instituto Oswaldo Cruz 108:590-595.

54. Nallapareddy SR, Weinstock, G.M., Murray, B.E. 2003. Clinical isolates of Enterococcus faecium exhibit strain-specific collagen binding mediated by Acm, a new member of the MSCRAMM family. Mol Microbiol 47:1733-1747.

55. Hendrickx AP, van Luit-Asbroek M, Schapendonk CM, van Wamel WJ, Braat JC, Wijnands LM, Bonten MJ, Willems RJ. 2009. SgrA, a nidogen-binding LPXTG surface adhesin implicated in biofilm formation, and $E c b A$, a collagen binding MSCRAMM, are two novel adhesins of hospital-acquired Enterococcus faecium. Infect Immun 77:5097-106. 
Genomic Epidemiology of VREfm in Victoria

56. Hendrickx AP, Bonten MJ, van Luit-Asbroek M, Schapendonk CM, Kragten AH, Willems RJ. 2008. Expression of two distinct types of pili by a hospital-acquired Enterococcus faecium isolate. Microbiology 154:3212-23.

57. Willems RJL, Homan W, Top J, van Santen-Verheuvel M, Tribe D, Manzioros X, Gaillard C, Vandenbroucke-Grauls CMJE, Mascini EM, van Kregten E, van Embden JDA, Bonten MJM. 2001. Variant esp gene as a marker of a distinct genetic lineage of vancomycin resistant Enterococcus faecium spreading in hospitals. Lancet 357:853855.

58. Leavis H, Top J, Shankar N, Borgen K, Bonten M, van Embden J, Willems RJL. 2004. A novel putative enterococcal pathogenicity island linked to the esp virulence gene of Enterococcus faecium and associated with epidemicity. J Bacteriol 186:672682.

59. Carter GP, Buultjens AH, Ballard SA, Baines SL, Tomita T, Strachan J, Johnson PD, Ferguson JK, Seemann T, Stinear TP, Howden BP. 2016. Emergence of endemic MLST non-typeable vancomycin-resistant Enterococcus faecium. J Antimicrob Chemother 71:3367-3371.

60. Christiansen K TJ, George N, Pearson J. on behalf of the Australian Group for Antimicrobial Resistance. 2005. Antimicrobial Susceptibility Report of Enterococcus Isolates from the Australian Group on Antimicrobial Resistance (AGAR): 2005 Surveillance Report.

61. Christiansen K TJ, Gottlieb, Bell J, George N, Pearson J. on behalf of the Australian Group for Antimicrobial Resistance. 2007. Enterococcus species Survey: 2007 Antimicrobial Susceptibility Report. 
Genomic Epidemiology of VREfm in Victoria

62. Christiansen K TJ, Gottlieb T, bell J, George N, Pearson J. on behalf of the Australian Group for Antimicrobial Resistance. 2009. Enterococcus species Survey: 2009 Antimicrobial Susceptibility Report.

63. Bell J, Turnidge J, Coombs G, O'Brien F. 1998. Emergence and epidemiology of vancomycin-resistant enterococci in Australia. Commun Dis Intell 22:249-52.

64. Worth LJ, Slavin MA, Vankerckhoven V, Goossens H, Grabsch EA, Thursky KA. 2008. Virulence determinants in vancomycin-resistant Enterococcus faecium vanB: clonal distribution, prevalence and significance of esp and hyl in Australian patients with haematological disorders. J Hosp Infect 68:137-44.

65. Yang J, Jiang Y, Guo L, Ye L, Ma Y, Luo Y. 2016. Prevalence of diverse clones of vancomycin-resistant Enterococcus faecium ST78 in a Chinese hospital. Microb Drug Resist 22:294-300.

66. Ruzon FI, de Paula SB, Kanoshiki RL, Pereira-Santos J, Kerbauy G, Kobayashi RK, Yamauchi LM, Perugini MR, Yamada-Ogatta SF. 2010. Virulence determinants in vancomycin-resistant Enterococcus faecium vanA isolated from different sources at University Hospital of Londrina, Parana, Brazil. J Microbiol 48:814-21.

67. Brodrick HJ, Raven KE, Harrison EM, Blane B, Reuter S, Torok ME, Parkhill J, Peacock SJ. 2016. Whole-genome sequencing reveals transmission of vancomycinresistant Enterococcus faecium in a healthcare network. Genome Med 8:4.

68. Pinholt M, Larner-Svensson H, Littauer P, Moser CE, Pedersen M, Lemming LE, Ejlertsen T, Sondergaard TS, Holzknecht BJ, Justesen US, Dzajic E, Olsen SS, Nielsen JB, Worning P, Hammerum AM, Westh H, Jakobsen L. 2015. Multiple hospital outbreaks of vanA Enterococcus faecium in Denmark, 2012-13, investigated by WGS, MLST and PFGE. J Antimicrob Chemother 70:2474-82. 
Genomic Epidemiology of VREfm in Victoria

69. Lam MM, Seemann T, Bulach DM, Gladman SL, Chen H, Haring V, Moore RJ,

Ballard S, Grayson ML, Johnson PD, Howden BP, Stinear TP. 2012. Comparative

analysis of the first complete Enterococcus faecium genome. J Bacteriol 194:2334-41.

70. Weigel LM, Clewell DB, Gill SR, Clark NC, McDougal LK, Flannagan SE, Kolonay

JF, Shetty J, Killgore GE, Tenover FC. 2003. Genetic analysis of a high-level

vancomycin-resistant isolate of Staphylococcus aureus. Science 302:1569-71. 
bioRxiv preprint doi: https://doi.org/10.1101/289975; this version posted March 28, 2018. The copyright holder for this preprint (which was not certified by peer review) is the author/funder, who has granted bioRxiv a license to display the preprint in perpetuity. It is made available under aCC-BY-NC-ND 4.0 International license.

Figure 1. Specimen type by van gene for Victorian E. faecium isolates

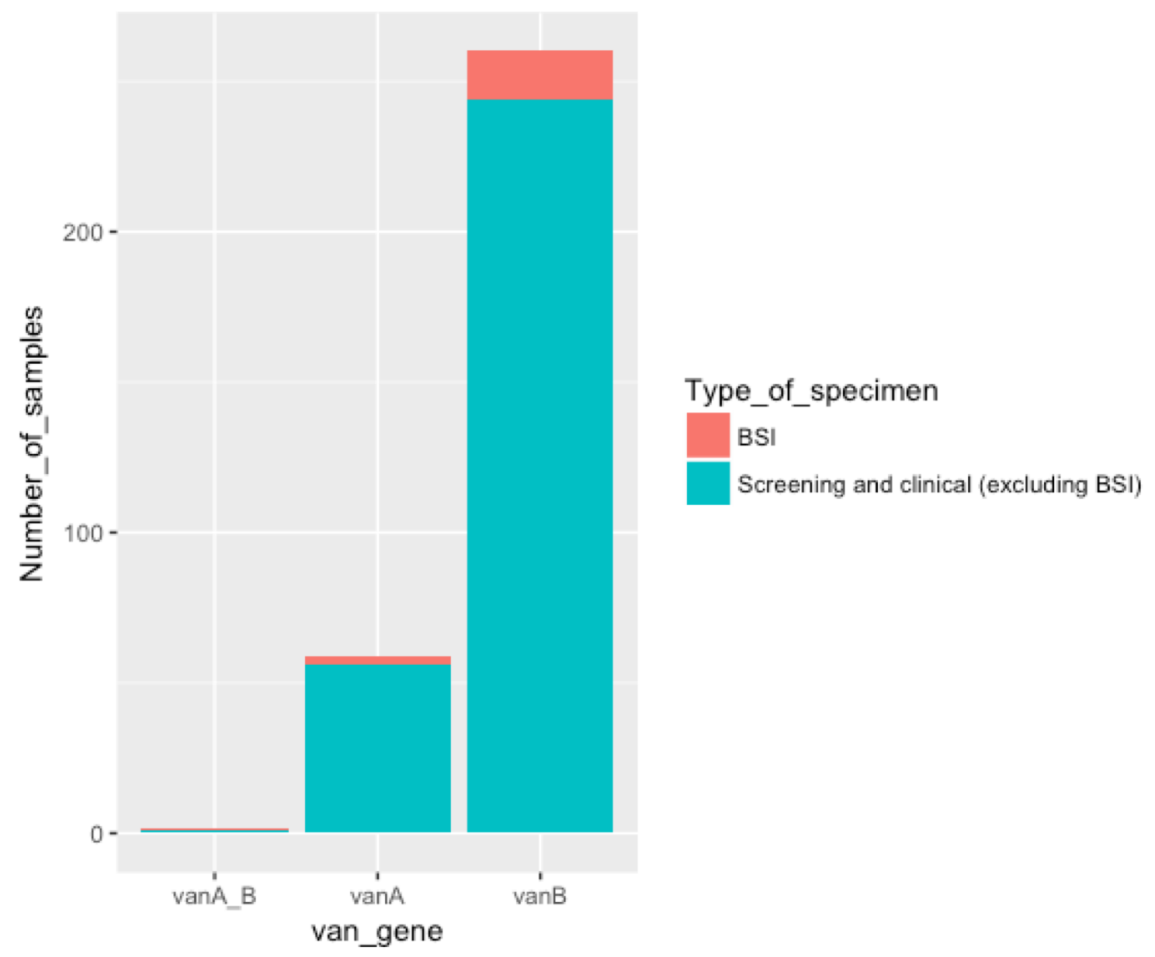

Legend. Only 2 isolates were $v a n A+v a n B$; only one was from a blood-stream infection (BSI). As all 10 VSEfm isolates were from BSI by design, these have not been shown. Fisher Exact test comparing the proportion of BSI among isolates with vanA vs vanB: $\mathrm{p}=0.52$. 
bioRxiv preprint doi: https://doi.org/10.1101/289975; this version posted March 28,2018 . The copyright holder for this preprint (which was not certified by peer review) is the author/funder, who has granted bioRxiv a license to display the preprint in perpetuity. It is made available under aCC-BY-NC-ND 4.0 International license.

Figure 2. Maximum likelihood tree of E. faecium in Victoria
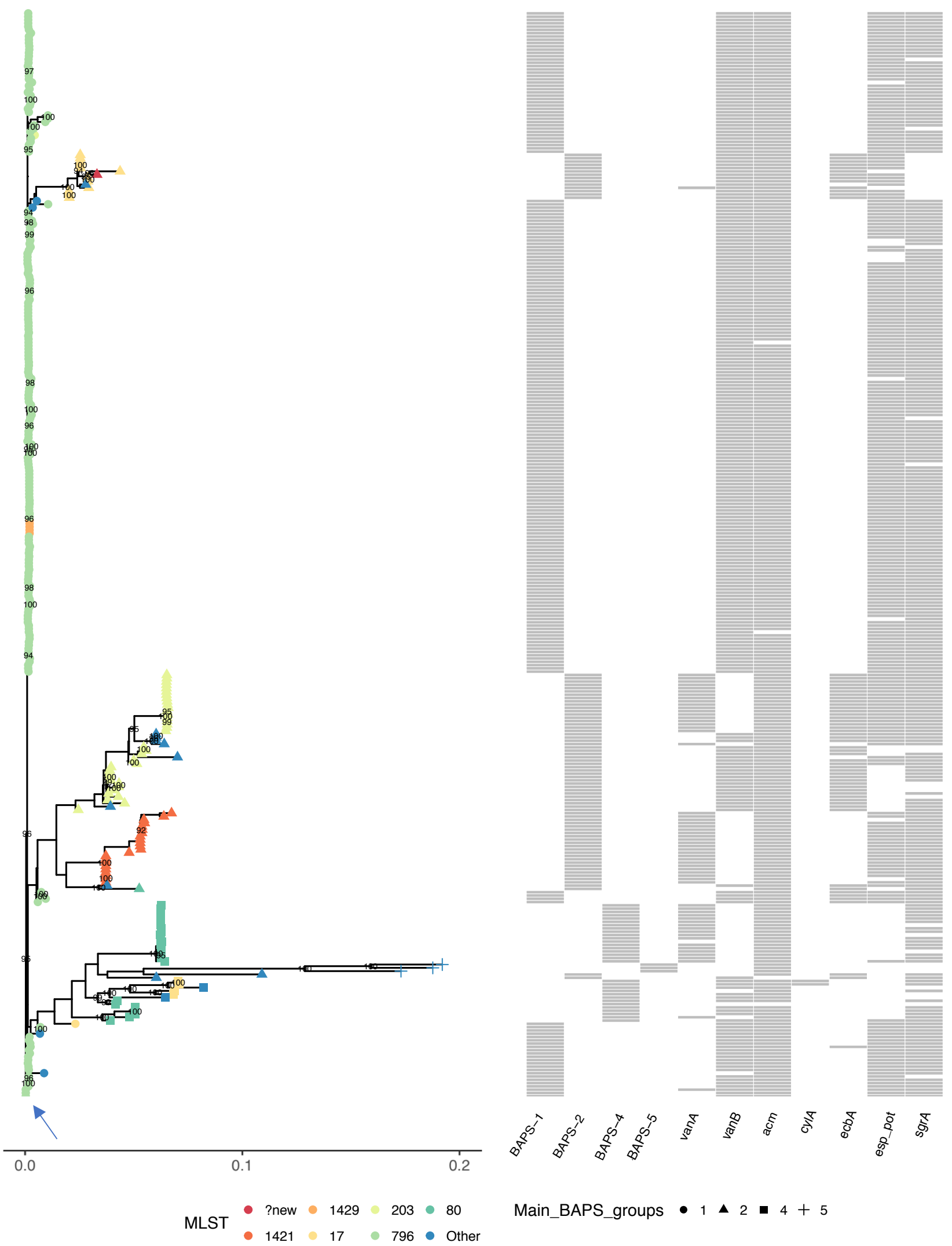

Legend. 6,497 recombination-adjusted core SNPs from all 331 isolates (321 VREfm and 10 VSEfm) were concatenated, and used to produce a maximum likelihood tree in RAxML (36) under a General Time Reversible model with gamma rate heterogeneity. One thousand bootstrap replicates were performed to assess confidence in the phylogeny. BAPS-3 is not shown; AUSMDU00004157 (an ST54 VSEfm) was the only isolate in this clade, and was $>3,800$ SNPs from all other isolates, so it was excluded from this figure for easier visualization. Branches with bootstrap support $>90$ are shown. Many branches had very low bootstrap support, thus hierarchical Bayesian Analysis of Population Structure (hierBAPS) was used to further validate the clusters identified. Main BAPS groups are shown. The reference is identified with an arrow. No isolates had ebpA, hyl, gelE, agg, pilA or pilB, therefore these genes have not been shown. 
bioRxiv preprint doi: https://doi org/10.1101/289975; this version posted March 28, 2018. The copyright holder for this preprint (which was not certified by peer review) is the author/funder, who has granted bioRxiv a license to display the preprint in perpetuity. It is made available under aCC-BY-NC-ND 4.0 International license.

Figure 3. Recombination-adjusted pairwise core SNPs separating Victorian isolates

Panel A. Overall pairwise comparisons, including isolates with both vanA and vanB and VSEfm

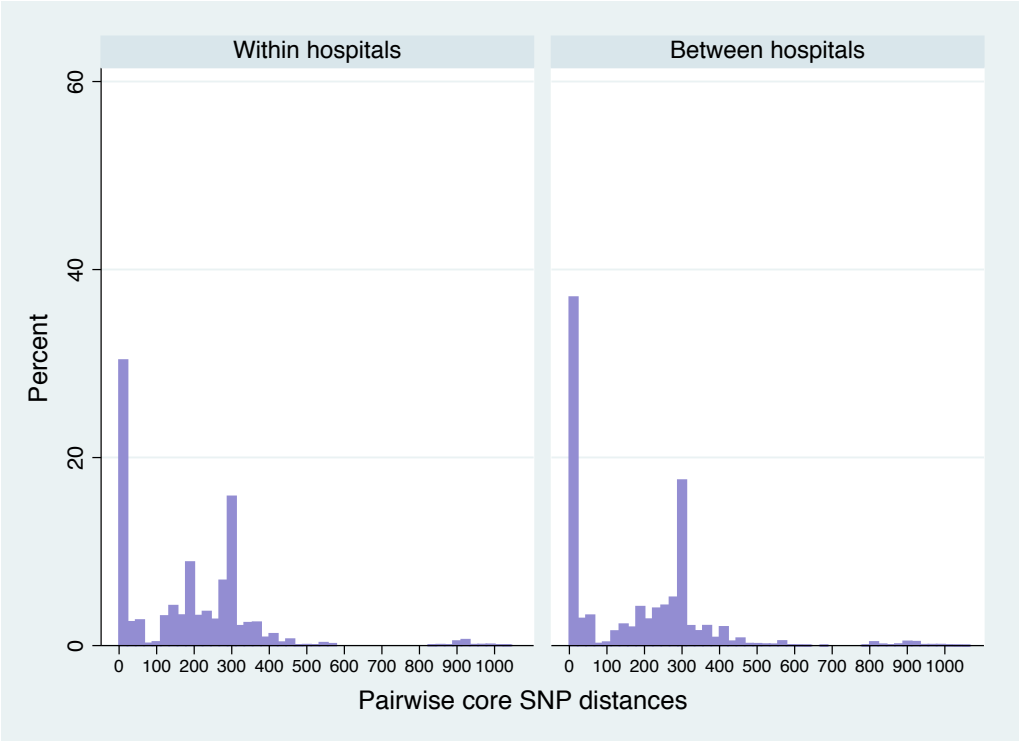

Panel B. Pairwise comparisons between vanA-only isolates

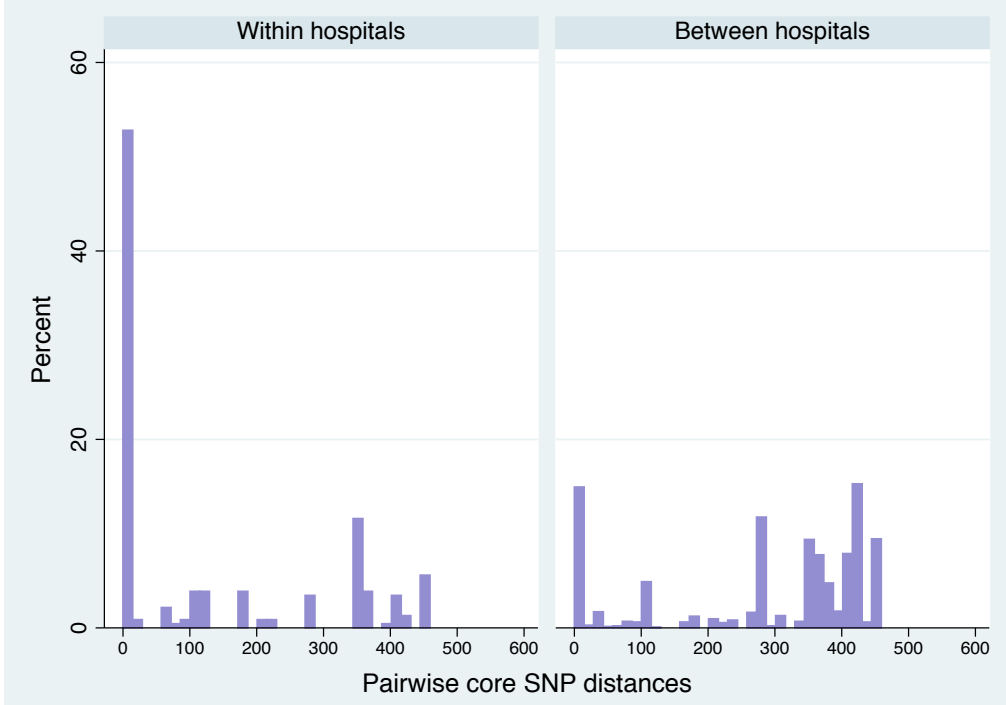




\section{Panel C. Pairwise comparisons between vanB-only isolates}

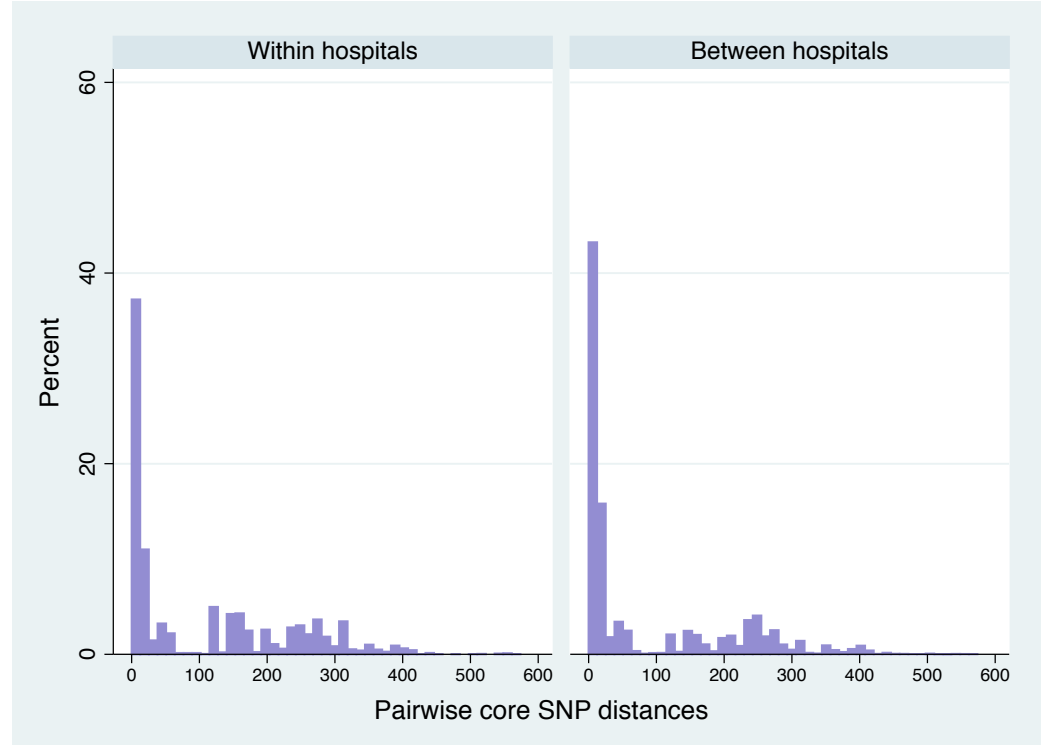

Legend. A. Pairwise SNP comparisons are shown regardless of van gene. There were 54,567 total pairwise comparisons, after excluding those involving isolates with missing data on hospital/HCN, those from unknown clinics, and pairs within the same patients.

Between hospitals, the median pairwise SNPs was 193 (IQR 11-298), while within hospitals, the median was 196 (IQR 13-297). For easier visualization, as one VSEfm isolate (AUSMDU00004157) was a minimum of 3,893 SNPs from all others, only comparisons between the 330 other isolates are shown in the figure.

B. Pairwise comparisons between isolates with vanA only are shown. There were 1,706 such comparisons (233 within hospitals 1,473 between hospitals). The median pairwise SNPS within hospitals was 10 (IQR 1-357) compared to a median of 356 (IQR 179-416) between hospitals ( $\mathrm{p}=0.0001$ ).

C. Pairwise comparisons between isolates with $v a n B$ only are shown. There were 33,639 such comparisons $(3,104$ within hospitals, 30,528 between hospitals). The median pairwise SNPs within hospitals was 40 (IQR 9-206), compared to a median of 15 (IQR 8-174) between hospitals $(\mathrm{p}=0.0001)$.

Isolates that were both $v a n A$ and $\operatorname{van} B$-positive $(\mathrm{n}=2)$, and isolates that were $\operatorname{van} A$ and $\operatorname{van} B$-negative $(\mathrm{n}=10)$ were excluded from Panels B and C. 
bioRxiv preprint doi: https://doi.org/10.1101/289975; this version posted March 28, 2018. The copyright holder for this preprint (which was not certified by peer review) is the author/funder, who has granted bioRxiv a license to display the preprint in perpetuity. It is made available under Figure 4. Homology between vanA-harbouring plasmids

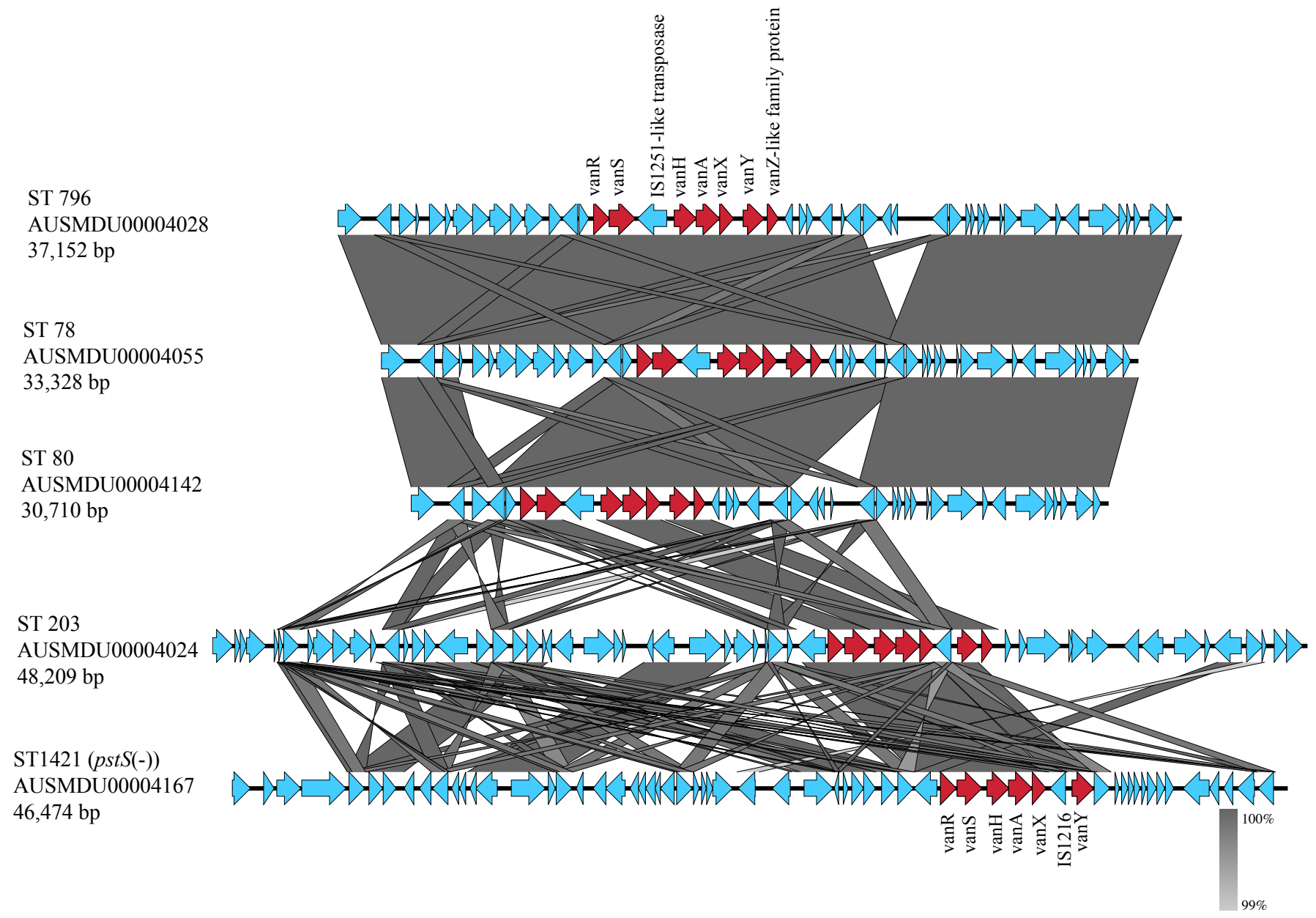

Legend. PacBio Single Molecule Real-Time sequencing was performed on a vanA-positive isolate from each of the main vanA-associated STs (ST 78, ST80, 203, 796, and ST1421). Plasmids harbouring vanA were initially identified using ABRicate (https://github.com/tseemann/abricate), and subsequently annotated using the Enterococcus database (https://github.com/tseemann/prokka/blob/master/db/genus/Enterococcus). This figure was produced using EasyFig (v. 2.2.2, version); blastn was used to compare sequence homology. Only hits with a minimum length of $200 \mathrm{bp}$ and at least 99\% identity are shown for clarity. van genes are indicated in red, all other genes are indicated in blue. 